\title{
A Prospective Clinical Study on the Impact of Occupation on Inguinodynia after Inguinal Hernia Repair
}

\section{Ojas Vijayanand Potdar, Nitin Borle*, Sanjay Nagral, Shraddha Bhone and Amrita Patkar}

Department of General Surgery, K. B. Bhabha Municipal General Hospital, Mumbai, India

*Corresponding author: Nitin Borle, Department of General Surgery, K. B. Bhabha Municipal General Hospital, Mumbai, India, Tel: +91-9619915003; E-mail: drnitinborle@gmail.com

Received date: August 24, 2018; Accepted date: September 5, 2018; Published date: September 11, 2018

Copyright: (C) 2018 Potdar OV, et al. This is an open-access article distributed under the terms of the Creative Commons Attribution License, which permits unrestricted use, distribution and reproduction in any medium, provided the original author and source are credited.

\begin{abstract}
Inguinodynia is defined as groin pain at or beyond 3 months following inguinal hernia repair. There is limited literature on this problem from India. Studies have investigated role of different risk factors for inguinodynia like mesh, inguinal nerves identification during surgery, technique of repair etc. However, to our knowledge there is no study on the impact of occupation on inguinodynia.
\end{abstract}

Objective: This study attempts to study the role of occupation in incidence of inguinodynia following surgery.

Methods: A prospective study was conducted at a secondary care hospital in Mumbai. 152 adult patients with uncomplicated inguinal hernias who underwent repair by open or laparoscopic technique were enrolled over 2 years. Patients were preoperatively questioned regarding the nature of their occupation and presence of pain associated with inguinal hernia, then divided into five occupation classes depending upon amount of weight lifting involved in their occupation. Postoperatively, they were followed up for 6 months and assessed for inguinodynia by a structured questionnaire. Incidence of inguinodynia was then compared between the different occupation classes.

Results: The incidence of inguinodynia at the end of 3rd and 6th month follow-up was $12.10 \%$ (19/157 surgeries) and $10.19 \%$ (16/157 surgeries) respectively. Incidence of inguinodynia increased from $8.8 \%$ for a sedentary worker to $38.5 \%$ for an extremely heavyweight worker at 6 months follow-up. By Pearson's Chi-Square test, the p-value was 0.003 which is statistically significant.

Conclusion: Thus, the incidence of inguinodynia increased as amount of weight lifting at work increased, suggesting a role of occupation in inguinodynia.

Keywords: Inguinal Hernia; Pain; Prospective study; Occupation

\section{Introduction}

Chronic groin pain (Inguinodynia) following inguinal hernia repair is a significant problem worldwide with the incidence in world literature being about 7\%-12\% [1]. Moderate to severe pain persisting more than 3 months after inguinal hernioplasty should be considered as pathological. The major reasons for inguinodynia have been identified as neuropathic causes due to inguinal nerves damage or non-neuropathic causes due to mesh or other related factors.

\section{Review of Literature}

The symptom complex of inguinodynia varies from a dull ache to sharp shooting pain or pricking or burning pain along the distribution of inguinal nerves. Thorough history and meticulous clinical examination should be performed to identify the exact cause of inguinodynia. Various studies $[2,3]$ look at difference in inguinodynia rates with the use of mesh vs. non-mesh repair [4], use of heavyweight vs. lightweight mesh and mesh fixation with sutures vs. glue [5-11]. Though there is no convincing evidence favoring one over the other, lightweight meshes are generally preferred because of their lesser foreign body reaction and better tolerance by the patients.
Identification of all 3 nerves (i.e. ilioinguinal, iliohypogastric and genital branch of genitofemoral nerves) has been shown to be an important factor in reducing inguinodynia, though there are no well conducted randomised studies to recommend benefits of nerve excision vs. preservation [12-18].

Various methods to reduce the occurrence of inguinodynia have been suggested which include prophylactic ilioinguinal neurectomy, laparoscopic TEP repair, use of lightweight meshes and use of fibrin glue sealant for mesh fixation has been described [19-21].

There is limited literature on this problem from India. Also to our knowledge, there are no studies correlating incidence of inguinodynia with various occupation classes. We believe that it is important to recognize the incidence of this problem as the patients can be adequately counseled preoperatively. Also since many inguinal hernias are asymptomatic or minimally symptomatic, this issue becomes even more relevant [22-24].

Inguinodynia: The International Association for the Study of Pain (IASP) described inguinodynia as "groin pain reported by patient at or beyond 3 months following inguinal hernia repair" (3 months being normal healing time) [25]. Postoperative pain is defined as groin pain or discomfort within 3 months after operation [19]. 


\section{Methodology}

\section{Aim}

- To calculate the incidence of inguinodynia

- To study role of occupation in inguinodynia

- Study site: Secondary care hospital in Mumbai

- Study population: Patients above the age of 18 years with inguinal hernia who underwent inguinal hernia repair at the hospital

- Study design: Prospective Observational Study

- Sample size: A total of 152 patients (total of 159 inguinal hernia cases as 7 patients had bilateral inguinal hernias) were included

- Duration: 2 years (August 2015-July 2017)

Inclusion criteria: All Patients who underwent elective open or laparoscopic inguinal hernia repair surgery during the defined period.

\section{Exclusion criteria:}

1. Complicated and recurrent hernias

2. Children under 18 years of age

The patients enrolled into the study were assessed preoperatively and vital information was gathered regarding their personal profile including occupation and important clinical history regarding the type, duration of hernia and its reducibility; whether the patient had any preoperative symptoms and any co morbid conditions already diagnosed in the patients. The patients were divided into 5 occupation classes depending upon the amount of weight lifting involved in their occupation as described by Code of Federal Regulations, USA [26].

(a) Sedentary work: involves lifting no more than $5 \mathrm{~kg}$ at a time and occasionally lifting or carrying articles like docket files, ledgers, and small tools

(b) Light work: involves lifting no more than $9 \mathrm{~kg}$ at a time with frequent lifting or carrying of objects weighing up to $5 \mathrm{~kg}$

(c) Medium work: involves lifting no more than $22 \mathrm{~kg}$ at a time with frequent lifting or carrying of objects weighing up to $11 \mathrm{~kg}$

(d) Heavy work: involves lifting no more than $45 \mathrm{~kg}$ at a time with frequent lifting or carrying of objects weighing up to $22 \mathrm{~kg}$

(e) Very heavy work: involves lifting objects weighing more than 45 $\mathrm{kg}$ at a time with frequent lifting or carrying of objects weighing $22 \mathrm{~kg}$ or more

The patients underwent either open (Lichtenstein's Tension free mesh repair) or laparoscopic repair (Transabdominal Preperitoneal (TAPP) or Total Extra peritoneal (TEP) Repair). The decision for open or laparoscopic repair was based on type of hernia, fitness of patient and surgeon preferences. The surgeries were carried out by Resident doctors and senior surgeons. Both open and laparoscopic procedures were standardized in terms of technique, type of mesh and type of fixation of mesh.

Open technique: During surgery, attempts were made to identify all the 3 nerves: Ilioinguinal, iliohypogastric and genital branch of genitofemoral nerves and to preserve them. Accidental injuries to any of the nerves or failure to identify all 3 nerves were mentioned in the operative notes. If accidental nerve injury, the neurectomy was done. A heavyweight mesh $\left(82 \mathrm{gm} / \mathrm{m}^{2}\right)$ of size $7.5 \mathrm{~cm}$ by $15 \mathrm{~cm}$ was used.

Laparoscopic technique: During the repairs (TAPP/TEP) heavyweight mesh was used and fixed with tackers avoiding triangle of pain. The patients were called for follow up at $1^{\text {st }}$ week 1, 3, 6 months after surgery.

On follow up, the questionnaire elaborated in the study performa were filled by interviewing the patients. Those patients who did not follow up on personal meetings were contacted by telephonic interview. The assessment of pain in the follow up period was made by using visual analogue scale (Figure 1) [27].

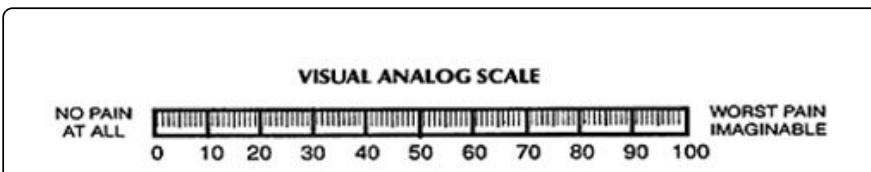

Figure 1: Visual Analog Scale; 0-4 mm: no pain, 5-44 mm: mild pain, $45-74 \mathrm{~mm}$ : moderate pain, $75-100 \mathrm{~mm}$ : Severe pain.

The diagnosis of inguinodynia is purely clinical. During follow up period; those patients who complained of pain were confirmed by clinical examination to elicit pain along the distribution of the affected nerve. Careful history to rule out anxiety and other psychosomatic complaints and accurate clinical examination helped to confirm inguinodynia related to surgery.

\section{Statistical methods: Data was entered in Microsoft Excel sheet.}

Data analysis was done using appropriate statistical software to calculate:

- Incidence of inguinodynia at $3^{\text {rd }}$ month and $6^{\text {th }}$ month follow-up

- To compare the incidences of inguinodynia amongst different occupation classes after inguinal hernia repair and statistically prove that the class of occupation has a role in inguinodynia

Comparison was done with Pearson's Chi-Square test as per results of Normality test. $\mathrm{P}<0.05$ taken as significant level.

\section{Results}

During the course of study, 152 patients were enrolled into the study. Of which 7 patients had bilateral hernias; so a total of 159 inguinal hernia repairs have been taken into account. Out of total of 159 surgeries; 140 were open repairs and 19 were laparoscopic repairs (17 TEP repairs and 2 TAPP repairs). Out of 159 surgeries, 157 were conducted on male patients and only 2 were conducted on female patients. The patients were divided into 5 occupation classes based upon the amount of weight lifting involved in their occupation. Preoperatively, out of 159 surgeries, only 27 were associated with pain; out of which only 7 had severe pain (Table 1).

\begin{tabular}{|l|l|l|}
\hline Class of occupation & Frequency & Percent \\
\hline Sedentary Worker & 93 & $58.49 \%$ \\
\hline Light weight & 32 & $20.13 \%$ \\
\hline Medium weight & 10 & $6.29 \%$ \\
\hline Heavy weight & 11 & $6.92 \%$ \\
\hline Extremely heavy weight & 13 & $8.18 \%$ \\
\hline Total & 159 & $100.00 \%$ \\
\hline
\end{tabular}

Table 1: Distribution of cases as per class of occupation. 
Citation: Potdar OV, Borle N, Nagral S, Bhone S, Patkar A (2018) A Prospective Clinical Study on the Impact of Occupation on Inguinodynia after Inguinal Hernia Repair. Surgery Curr Res 8: 313. doi:10.4172/2161-1076.1000313

Page 3 of 5

In 159 surgeries, ilioinguinal nerve was identified in 140 surgeries; out of which it was preserved in 131 cases and resected in the remaining 9 cases. Iliohypogastric nerve was identified in 126 surgeries and out of which it was preserved in 122 surgeries, resected in 4 cases and not identified in 33 cases.

Genital branch of genitofemoral nerve was identified and preserved only in 26 cases and not identified in 133 cases. The patients were asked to follow up at 1 st week, 1 st month, $3^{\text {rd }}$ month and $6^{\text {th }}$ month. There was no data lost to follow up at 1st week follow up. Out of 159 surgeries; 48 patients (since 5 patients had bilateral hernias; so 53 cases) reported pain in the inguinal region.

At $1^{\text {st }}$ month follow-up; 2 patients were lost to follow-up. 29 patients reported pain in the inguinal region. Out of 29 patients, 4 patients had operated inguinal hernia on both sides who complained of pain on both sides; so a total of 33 cases reported pain in inguinal region.

At $3^{\text {rd }}$ month follow-up; 2 patients were lost to follow-up. 18 patients reported pain in inguinal region which was classical of inguinodynia. Out of 18 patients, one patient had inguinal hernia operated on both sides and complained of pain on both sides; so a total of 19 cases reported pain in the inguinal region at $3^{\text {rd }}$ month follow-up. Therefore, the incidence of pain was $12.10 \%$ at $3^{\text {rd }}$ month follow-up; of which 15 cases had mild pain and 4 cases had moderate pain.

At $6^{\text {th }}$ month follow-up 2 patients were lost to follow-up. 15 patients reported pain in inguinal region which was of classical of inguinodynia. Out of these 15 patients; 1 patient had inguinal hernia operated on both sides and complained of pain on both sides. Therefore, the incidence of inguinodynia at $6^{\text {th }}$ month follow-up was 10.19\%; of which 13 cases had mild pain and 3 cases had moderate pain.

At $3^{\text {rd }}$ month follow up, it was found that incidence of inguinodynia increased as amount of weight lifting at work increased from being a sedentary worker (incidence-9.9\%) to being an extremely heavy weight worker (incidence-38.5\%) except in case of light weight worker where the incidence of inguinodynia was 3.1\%. After applying Pearson's ChiSquare test, $\mathrm{p}$-value was $0.008(\mathrm{P}<0.05)$, hence, this association was statistically significant (Tables 2 and 3 ).

\begin{tabular}{|c|c|c|c|c|}
\hline \multirow{2}{*}{ Occupation Class } & & \multicolumn{2}{|c|}{$\begin{array}{l}\text { Inguinodynia at } 3 \mathrm{rd} \\
\text { month }\end{array}$} & \multirow{3}{*}{$\begin{array}{l}\text { Total } \\
91\end{array}$} \\
\hline & & Yes & No & \\
\hline \multirow{2}{*}{ Sedentary } & No. & 9 & 82 & \\
\hline & $\%$ & $9.90 \%$ & $90.10 \%$ & $100.00 \%$ \\
\hline \multirow{2}{*}{ Light weight } & No. & 1 & 31 & 32 \\
\hline & $\%$ & $3.10 \%$ & $96.90 \%$ & $100.00 \%$ \\
\hline \multirow{2}{*}{ Medium weight } & No. & 1 & 9 & 10 \\
\hline & $\%$ & $10.00 \%$ & $90.00 \%$ & $100.00 \%$ \\
\hline \multirow{2}{*}{ Heavy weight } & No. & 3 & 8 & 11 \\
\hline & $\%$ & $27.30 \%$ & $72.70 \%$ & $100.00 \%$ \\
\hline \multirow{2}{*}{ Extremely heavy weight } & No. & 5 & 8 & 13 \\
\hline & $\%$ & $38.50 \%$ & $61.50 \%$ & $100.00 \%$ \\
\hline Total & No. & 19 & 138 & 157 \\
\hline
\end{tabular}

\begin{tabular}{|l|l|l|l|l|}
\hline & $\%$ & $12.10 \%$ & $87.90 \%$ & $100.00 \%$ \\
\hline
\end{tabular}

Table 2: Comparison of incidence of inguinodynia amongst different occupation classes at $3^{\text {rd }}$ month follow-up.

\begin{tabular}{|l|l|l|l|l|}
\hline Chi-Square test & Value & df & p-value & Association \\
\hline Pearson Chi-Square & 13.756 & 4 & 0.008 & Significant \\
\hline
\end{tabular}

Table 3: Pearson's Chi-Square test at $3{ }^{\text {rd }}$ month follow-up.

At $6^{\text {th }}$ month follow-up, it was found that incidence of inguinodynia increased as amount of weight lifting at work increased from being a sedentary worker (incidence-8.8\%) to being an extremely heavy weight worker (incidence-38.5\%) except in case of light weight worker; where the incidence of inguinodynia was zero percent. After applying Pearson's Chi-Square test, $p$-value was ] $0.003(p<0.05)$, hence, this association was statistically significant (Tables 4 and 5).

\begin{tabular}{|c|c|c|c|c|}
\hline \multirow{2}{*}{ Occupation Class } & & \multicolumn{2}{|c|}{$\begin{array}{l}\text { Inguinodynia at } 6 \text { th } \\
\text { month }\end{array}$} & \multirow[t]{2}{*}{ Total } \\
\hline & & Yes & No & \\
\hline \multirow{2}{*}{ Sedentary } & No. & 8 & 83 & 91 \\
\hline & $\%$ & $8.80 \%$ & $91.20 \%$ & $100.00 \%$ \\
\hline \multirow{2}{*}{ Light weight } & No. & 0 & 32 & 32 \\
\hline & $\%$ & $0.00 \%$ & $100.00 \%$ & $100.00 \%$ \\
\hline \multirow{2}{*}{ Medium weight } & No. & 1 & 9 & 10 \\
\hline & $\%$ & $10.00 \%$ & $90.00 \%$ & $100.00 \%$ \\
\hline \multirow{2}{*}{ Heavy weight } & No. & 2 & 9 & 11 \\
\hline & $\%$ & $18.20 \%$ & $81.80 \%$ & $100.00 \%$ \\
\hline \multirow{2}{*}{ Extremely heavy weight } & No. & 5 & 8 & 13 \\
\hline & $\%$ & $38.50 \%$ & $61.50 \%$ & $100.00 \%$ \\
\hline \multirow{2}{*}{ Total } & No. & 16 & 141 & 157 \\
\hline & $\%$ & $10.20 \%$ & $89.80 \%$ & $100.00 \%$ \\
\hline
\end{tabular}

Table 4: Comparison of incidence of inguinodynia amongst different occupation classes at $6^{\text {th }}$ month follow-up.

\begin{tabular}{|l|l|l|l|l|}
\hline Chi-Square test & Value & df & p-value & Association \\
\hline Pearson Chi-Square & 13.756 & 4 & 0.008 & Significant \\
\hline
\end{tabular}

Table 5: Pearson's Chi-Square test at $6^{\text {th }}$ month follow-up.

\section{Discussion}

Most of inguinal hernias are asymptomatic or minimally symptomatic. Various factors have been studied to have a role in incidence of inguinodynia. We have made an attempt to study the role of occupation in inguinodynia. Inguinodynia is defined as groin pain reported by patient at or beyond 3 months following inguinal hernia repair [25]. In this study, patients were assessed preoperatively for 
presence of pain in inguinal region. The patients were divided into 5 occupation classes based upon amount of weight lifting at work.

In studies by Kehlet $\mathrm{H}$ and Franneby $\mathrm{U}$ et al. number of factors have been studied to play a role in the inguinodynia which include younger age, history of preoperative pain, interval $<3$ years from prior surgery $[2,3]$, severe early postoperative pain, postoperative complications, postoperative sensory disorder, female sex, ilioinguinal nerve excision, iliohypogastric nerve excision, anterior hernia repair and recurrent hernia repair. Jeroukhimov I in his study discussed about role of nonabsorbable sutures to anchor the mesh to play a role in reduced incidence of inguinodynia [5].

Barazanchi AW et al. studied the role of routine ilioinguinal and iliohypogastric neurectomy during the inguinal hernia repair [14]. It was found that routine ilioinguinal neurectomy during Lichtensteintype herniorrhaphy seems to be a safe and effective method to reduce pain in short and midterm period, but may have little long-term impact. Iliohypogastric neurectomy seems to reduce pain in at least the short term postoperative period. In our study, an attempt was made to identify and preserve all 3 inguinal nerves during repair. Resection was only done when unavoidable.

At $3^{\text {rd }}$ month follow-up of 157 cases, only 1 in 9 cases in which ilioinguinal nerve was identified and resected reported pain. However, role of ilioinguinal nerve identification to reduce incidence of inguinodynia at $3^{\text {rd }}$ month follow-up was not statistically significant. For iliohypogastric nerve, 4 cases in which it was identified and resected; none reported pain. However, the role of iliohypogastric nerve identification to reduce incidence of inguinodynia at $3^{\text {rd }}$ month follow-up was not statistically significant.

At 6th month follow-up of 157 cases; 9 cases with ilioinguinal nerve was identified and resected; none reported pain. However, role of ilioinguinal nerve identification to reduce incidence of inguinodynia at $6^{\text {th }}$ month follow up was not statistically significant. In case of iliohypogastric nerve, 4 cases with nerve resection, none reported pain. However, the role of iliohypogastric nerve identification to reduce the incidence of inguinodynia at $6^{\text {th }}$ month follow-up was not statistically significant. Also in those cases (19/157 cases) who reported pain in inguinal region at $3^{\text {rd }}$ month follow-up, all except 2 had ilioinguinal nerve identified and preserved; and all except 3 had iliohypogastric nerve identified and preserved.

At $6^{\text {th }}$ month follow-up (16/157 cases) who reported pain in inguinal region, all except one had ilioinguinal nerve identified and preserved; and all except 3 had iliohypogastric nerve was identified and preserved.

In our study, at $3^{\text {rd }}$ month follow-up; 2 patients were lost to followup, so 150 patients reported for follow-up patients had inguinodynia [18]. Out of 18 patients, one patient had bilateral repair and complained of pain bilaterally. Therefore, incidence of pain was $12.10 \%$ at $3^{\text {rd }}$ month follow-up, 15 cases with mild pain and 4 cases with moderate pain.

At $6^{\text {th }}$ month follow-up; 2 patients were lost to follow-up. 15 patients reported pain the inguinal region. Out of 15 patients, 1 patient had bilateral repair and complained of pain bilaterally. Therefore, incidence of inguinodynia at $6^{\text {th }}$ month follow-up was $10.19 \%, 13$ cases had mild pain and 3 cases had moderate pain. To our knowledge; there are no studies relating role of occupation in inguinodynia. This study aims to study role of occupation in incidence of inguinodynia.
At $3^{\text {rd }}$ month follow up, incidence of inguinodynia increased as amount of weight lifting involved in their occupation increased from being a sedentary worker (incidence-9.9\%) to being an extremely heavy weight worker (incidence-38.5\%). After applying Pearson's ChiSquare test, $\mathrm{p}$-value was $0.008(\mathrm{p}<0.05)$, hence, this association was statistically significant. However, the incidence of inguinodynia amongst lightweight workers is $3.1 \%$.

At $6^{\text {th }}$ month follow-up, it was observed that incidence of inguinodynia increased as amount of weight lifting at work increased from being a sedentary worker (incidence- $8.8 \%$ ) to being an extremely heavy weight worker (incidence-38.5\%). After applying Pearson's chisquare test, $\mathrm{p}$-value was $0.003(\mathrm{p}<0.05)$; hence, this association was statistically significant. However, incidence of inguinodynia amongst lightweight workers was found to be $0 \%$. Thus our study reveals that class of occupation may play a role in incidence of inguinodynia amongst patients undergoing hernia repair.

\section{Conclusion}

The incidence of inguinodynia in this study after inguinal hernia repair at $3 \mathrm{rd}$ and $6^{\text {th }}$ month follow-up was $12.10 \%$ and $10.19 \%$ respectively. The incidence of inguinodynia after inguinal hernia repair increases as the amount of weight lifting involved in their occupation increases from being a sedentary worker (incidence-9.9\% at $3^{\text {rd }}$ month and $8.8 \%$ at $6^{\text {th }}$ month) to being an extremely heavy weight worker (incidence-38.5\% at $3^{\text {rd }}$ month and $6^{\text {th }}$ month). Thus, class of occupation may play a role in incidence of inguinodynia in postoperative period amongst patients undergoing inguinal hernia repair.

Though the study has made has made a genuine attempt to establish the role of occupation in inguinodynia; a comparison study to confirm the role by advising patients to decrease the amount of weightlifting in their work in the postoperative period and look for its effect in the reduction of incidence of inguinodynia needs to be addressed.

\section{Recommendations}

Patients should be preoperatively counseled regarding the risk of inguinodynia and they may be counseled to consider decreasing the amount of weight lifting at work if possible to prevent inguinodynia.

\section{Limitations of the Study}

Most surgeries were performed by trainees, hence there may be some lapses in technique of inguinal hernia repair owing to their lack of expertise; however they were supervised by senior surgeons. The randomization of patients to either open or laparoscopic repairs was not done and it was based only on patient's preference and senior experienced surgeon's availability for laparoscopic repair.

\section{Conflict of Interest}

\section{References}

1. Niccolai P, Ouchchane L, Libier M, Beouche F, Belon M, et al. (2015) Persistent neuropathic pain after inguinal herniorrhaphy depending on the procedure (open mesh vs. Laparoscopy): A propensity-matched analysis. Can J Surg 58: 114-120. 
Citation: Potdar OV, Borle N, Nagral S, Bhone S, Patkar A (2018) A Prospective Clinical Study on the Impact of Occupation on Inguinodynia after Inguinal Hernia Repair. Surgery Curr Res 8: 313. doi:10.4172/2161-1076.1000313

Page 5 of 5

2. Kehlet H, Jensen TS, Woolf CJ (2006) Persistent postsurgical pain: Risk factors and prevention. Lancet 367: 1618-1625.

3. Franneby U, Sandblom G, Nordin P, Nyren O, Gunnarsson U, et al. (2006) Risk factors for long-term pain after hernia surgery. Ann Surg 244: 212-219.

4. Heise CP, Starling JR (1998) Mesh inguinodynia: A new clinical syndrome after inguinal herniorrhaphy? J Am Coll Surg 187: 514-518.

5. Jeroukhimov I, Wiser I, Karasic E, Nesterenko V, Poluksht N, et al. (2014) Reduced postoperative chronic pain after tension-free inguinal hernia repair using absorbable sutures: A single-blind randomized clinical trial. J Am Coll Surg 218: 102-110.

6. Benizrie I, Rahili A, Avallone S, Balestro JC, Cai J (2006) Open inguinal hernia repair by plug and patch: The value of fibrin sealant fixation. Hernia 10: 389-394.

7. Campanelli G, Pascual MH, Hoeferlin A, Rosenberg J, Champault G, et al. (2012) Randomized, controlled, blinded trial of Tisseel/Tissucol for mesh fixation in patients undergoing Lichtenstein technique for primary inguinal hernia repair: Results of the TIMELI trial. Ann Surg 255: 650-657.

8. Tolver MA, Rosenberg J, Juul P, Bisgaard T (2013) Randomized clinical trial of fibrin glue versus tacked fixation in laparoscopic groin hernia repair. Surg Endosc 27: 2727-2733.

9. Berney CR, Yeo AE (2013) Mesh fixation with fibrin sealant during endoscopic totally extra peritoneal inguinal hernia approach: A review of 640 repairs. Hernia 17: 709-717.

10. Shah NS, Fullwood C, Siriwardena AK, Sheen AJ (2014) Mesh fixation at laparoscopic inguinal hernia repair: A meta-analysis comparing tissue glue and tack fixation. World J Surg 38: 2558-2570.

11. Sun P, Cheng X, Deng S, Hu Q, Sun Y, et al. (2017) Mesh fixation with glue versus suture for chronic pain and recurrence in Lichtenstein inguinal hernioplasty. Cochrane Database Syst Rev 2: CD010814.

12. Hakeem A, Shanmugam V (2011) Inguinodynia following Lichtenstein tension-free hernia repair: A review. World J Gastroenterol 17: 1791-1796.

13. Wright RC, Sanders E (2011) Inguinal neuritis is common in primary inguinal hernia. Hernia 15: 393-398.

14. Barazanchi AW, Fagan PV, Smith BB, Hill AG (2016) Routine neurectomy of inguinal nerves during open onlay mesh hernia repair: A meta-analysis of randomized trials. Ann Surg 264: 64-72.
15. Aasvang EK, Mohl B, Kehlet H (2007) Ejaculatory pain: A specific post herniotomy pain syndrome? Anesthesiology 107: 298-304.

16. Reinpold WM, Nehls J, Eggert A (2011) Nerve management and chronic pain after open inguinal hernia repair: A prospective two phase study. Ann Surg 254: 163-168.

17. Hakeem A, Shanmugam V (2011) Current trends in the diagnosis and management of post-herniorrhaphy chronic groin pain. World J Gastrointest Surg 3: 73-81.

18. Alfieri S, Amid PK, Campanelli G, Izard G, Kehlet H, et al. (2011) International guidelines for prevention and management of postoperative chronic pain following inguinal hernia surgery. Hernia 15: 239-249.

19. Jaiswal Lt. Col SS, Chaudhry Brig R, Agrawal Maj A (2009) Chronic groin pain following Lichtenstein mesh hernioplasty for inguinal hernia. Is it a myth? Indian J Surg 71: 84-88,

20. Amid PK, Hiatt JR (2007) New understanding of the causes and surgical treatment of post herniorrhaphy inguinodynia and orchalgia. J Am Coll Surg 205: 381-385.

21. Chen D, Bjurstrom M, Amid P (2014) Pain control following inguinal herniorrhaphy: Current perspectives. J Pain Res 277-290.

22. Poobalan AS, Bruce J, King PM, Chambers WA, Krukowski ZH, et al. (2001) Chronic pain and quality of life following open inguinal hernia repair. Br J Surg 88: 1122-1126.

23. Kalliomaki ML, Sandblom G, Gunnarsson U, Gordh T (2009) Persistent pain after groin hernia surgery: A qualitative analysis of pain and its consequences for quality of life. Acta Anaesthesiol Scand 53: 236-246.

24. Cunningham J, Temple WJ, Mitchell P, Nixon JA, Preshaw RM, et al. (1996) Cooperative hernia study. Pain in the post repair patient. Ann Surg 224: 598-602.

25. Merskey H, Bogduk N (1994) Classification of chronic pain: Descriptions of chronic pain syndromes and definitions of pain terms. In Task Force on Taxonomy of the IASP. (2nd Edn) Seattle, WA: IASP Press pp: 209-214.

26. Article 404. 1567 Code of federal regulations, USA.

27. D Gould, Kelly D, Goldstone L, Gammon J (2001) Examining the validity of pressure ulcer risk assessment scales: developing and using illustrated patient simulations to collect the data. J Clin Nurs 10: 697-706. 